\title{
Dynamical constant mass-to-light ratio models of NGC 5128
}

\author{
S. Samurović
}

\author{
Astronomical Observatory, Volgina 7, 11160 Belgrade, Serbia \\ e-mail: srdjan@aob.bg.ac.rs
}

Received 25 November 2009 / Accepted 12 February 2010

\begin{abstract}
Context. The existence and amount of dark matter in early-type galaxies remains a hotly debated topic. We study dynamical models of NGC 5128, which do not include dark matter, to test the predictions of different constant mass-to-light models.

Aims. We use the measurements of the radial velocities of the planetary nebulae in NGC 5128 to test the predictions of Newtonian constant mass-to-light ratio models and we also extend our study to different MOND models.

Methods. The planetary nebulae of NGC 5128 were used as a tracer of the galaxy's gravitational potential. The Jeans equation was calculated for both the Newtonian mass-follows-light and the MOND approaches. Spherical symmetry is assumed and the calculations are performed for both isotropic and anisotropic cases.

Results. We solved the Jeans equation in spherical approximation and found that the isotropic Newtonian mass-follows-light models without dark matter may provide successful fits out to $6.4 R_{\mathrm{e}}$; to obtain a good fit in the outermost region $\left(\sim 10.7 R_{\mathrm{e}}\right)$ one needs either dark matter or tangential anisotropies. Concerning MOND models we found that no isotropic MOND model without dark matter can provide a successful fit interior to $\sim 6.4 R_{\mathrm{e}}$; for the anisotropic MOND models interior to $\sim 40$ arcmin only "standard" MOND model with tangential anisotropies can provide a successful fit of the observed velocity dispersion without the need of dark matter.
\end{abstract}

Key words. galaxies: elliptical and lenticular, $\mathrm{cD}$ - galaxies: kinematics and dynamics - galaxies: halos

\section{Introduction}

The problem of dark matter in early-type galaxies is still poorly understood. The reasons for this are numerous and are described in the literature (see e.g. Samurović 2007, for a review), so we here only briefly list several of the most important ones.

1) The luminosity of early-type galaxies in their outer parts is very low, which makes observations very difficult. Because of this only a very small number of early-type galaxies has been observed out to 3-4 effective radii to infer their internal kinematics $\left(R_{\mathrm{e}}\right.$ is the radius which encompasses half of the total light of a given galaxy; $3 R_{\mathrm{e}}$ contains $\sim 79$ per cent and $4 R_{\mathrm{e}}$ contains $\sim 85$ per cent of the total galactic light, according to the de Vaucouleurs law). Long-slit spectra used for obtaining internal kinematics are convenient because they also provide an information regarding stellar anisotropies, which is important for breaking the mass-anisotropy degeneracy (Tonry 1983). There are fortunately other methods that can be used for kinematical studies of early-type galaxies, and they are briefly given in the next two items.

2) X-rays studies (see Mathews \& Brighenti 2003a, for a review) are very useful because they can trace the gravitational potential out to large galactocentric distances $\left(R \gg 3 R_{\mathrm{e}}\right)$ without the aforementioned problem of anisotropies of orbits. The drawbacks also exist however: this methodology is based on the assumption of hydrostatic equilibrium, which has been disputed (e.g. Diehl \& Statler 2007), and not all early-type galaxies possess an X-ray halo (e.g. IC 3370, see Samurović \& Danziger 2005).

3) Different tracers of the gravitational potential like globular clusters (GCs) and planetary nebulae (PNe) are useful because they also yield results out to large galactocentric distances (again, $R \gg 3 R_{\mathrm{e}}$ ) (Romanowsky et al. 2003;
Schuberth et al. 2009). Note that the drawbacks here are also numerous: because one needs a large number of the tracers per bin, it is not possible to accurately determine the anisotropies of these objects, and one can have hints at most regarding the orbits of the tracers. The numbers of tracers also decreases in the outer parts of early-type galaxies, and these are the regions where dark matter is expected to dominate luminous matter. Additional problems may be seen when one uses GCs as tracers: there are two different populations, blue and red, for which the properties (like dispersion) may differ (see e.g. Richtler et al. 2004), thus reducing the number of tracers used per bin. For PNe all the observed objects are assumed to belong to one population.

A new, promising avenue to address the problem of dark matter in early-type galaxies is to use an integral-field unit to measure the line-of-sight velocity distribution (LOSVD) at large radii $\left(\sim 3-4 R_{\mathrm{e}}\right)$ (Weijmans et al. 2009 ).

So far the evidence of dark matter in early-type galaxies is inconclusive. Some galaxies appear to be devoid of it, or are dominated by certain types of orbits (the example of this class is NGC 3379, for which there is a lack of dark matter or dominance of radial orbits; see Romanowsky et al. 2003; Dekel et al. 2005), whereas some appear to possess huge massive dark halos (NGC 1399, see e.g. Samurović \& Danziger 2006; Schuberth et al. 2009). It is important to note that NGC 3379 is a relatively isolated object (part of the Leo I group) and NGC 1399 is a central galaxy of the Fornax cluster, which makes a direct comparison difficult.

The apparent lack of dark matter in NGC 3379 led researchers to initiate the study of early-type galaxies within the context of the MOND theory (Milgrom 1983). As was to be expected, the MOND models described NGC 3379 on all scales 
Table 1. Kinematics data for NGC 5128 for the sample of PNe.

\begin{tabular}{|c|c|c|c|c|c|c|c|}
\hline $\begin{array}{c}\langle r\rangle \\
(\operatorname{arcmin}) \\
(1)\end{array}$ & $\begin{array}{c}\sigma \\
\left(\mathrm{km} \mathrm{s}^{-1}\right) \\
(2)\end{array}$ & $\begin{array}{r}\text { err_o } \\
\left(\mathrm{km} \mathrm{s}^{-1}\right) \\
(3)\end{array}$ & $s_{3}$ & err_s $s_{3}$ & $s_{4}$ & err_s $s_{4}$ & $N$ \\
\hline 2 & 143 & 18 & -0.07 & 0.19 & -0.44 & 0.39 & 158 \\
\hline 6 & 134 & 7 & 0.29 & 0.17 & -0.02 & 0.34 & 204 \\
\hline 10 & 120 & 7 & -0.06 & 0.20 & 0.14 & 0.40 & 147 \\
\hline 14 & 128 & 11 & 0.44 & 0.29 & 0.16 & 0.58 & 72 \\
\hline 18 & 158 & 14 & -0.26 & 0.33 & -0.41 & 0.65 & 56 \\
\hline 22 & 99 & 12 & -0.56 & 0.43 & 0.80 & 0.85 & 33 \\
\hline 27 & 100 & 11 & 0.40 & 0.38 & 0.07 & 0.75 & 43 \\
\hline 35 & 89 & 10 & -0.31 & 0.38 & 0.83 & 0.76 & 42 \\
\hline 59 & 108 & 15 & -0.77 & 0.49 & -0.19 & 0.98 & 25 \\
\hline
\end{tabular}

Notes. Column (1): central point for a given bin; Col. (2): velocity dispersion for the sample of PNe in a given bin; Col. (3): formal errors for the velocity dispersion of the sample of PNe in a given bin; Col. (4): $s_{3}$ parameter for the sample of PNe in a given bin; Col. (5): formal errors for the $s_{3}$ parameter for the sample of PNe in a given bin; Col. (6): $s_{4}$ parameter for the sample of PNe in a given bin; Col. (7): formal errors for the $s_{4}$ parameter for the sample of PNe in a given bin; Col. (8): number of PNe in a given bin.

(Tiret et al. 2007). On the other hand, the MOND models failed to reproduce the kinematics of NGC 1399, and Richtler et al. (2008) concluded that the MOND models needed an "additional hypothetical dark halo", which of course makes MOND redundant. Samurović \& Ćirković (2008) studied NGC 4649 (M60), a giant elliptical galaxy in the Virgo cluster, and concluded that out to $\sim 5 R_{\mathrm{e}}$ the successful fit to the observed velocity dispersion can be obtained with the constant mass-to-light ratio in the $B$-band: $M / L_{B} \sim 7$ implying an absence or only a very low amount of dark matter in its outer parts. The MOND models with similar mass-to-light ratio also provided successful fits. Therefore, based on this admittedly small sample of early-type galaxies, one is led to conclude that when a galaxy needs dark matter to explain its kinematics (i.e. when a Newtonian approach fails), the MOND approach also has problems and appears to be insufficient. Vice versa, when Newtonian mass-follows-light models provide a successful description of the kinematics, the MOND models also appear to be correct. Note that the MOND models are also not insensitive to the mass-anisotropy degeneracy, because the Jeans equation, which needs to be solved, contains a term pertaining to the form of orbits (see below).

We study the galaxy NGC 5128, the nearest large elliptical galaxy, using different constant mass-to-light ratio models. The results of dynamical studies of NGC 5128 obtained so far suggest the existence of dark matter: Mathieu et al. (1996) used a quadratic programming method to infer that the mass-to-light ratio increases from the center and that about $\sim 50$ per cent of the galaxy is dark; Peng et al. (2004) also concluded that about 50 per cent of the galaxy is dark and that $M / L_{B} \sim 13$, but they note that the mass-to-light ratio they obtained is "much lower than what is typically expected for elliptical galaxy". Recently Łokas (2008) used the PNe data from Peng et al. (2004) and concluded that in the $V$-band, $M / L_{V}=26.3 \pm 16.9$, which in the $B$-band becomes $M / L_{B}=36.3 \pm 23.3$ (note the large error bars). We use $\mathrm{PNe}$ as tracers of the gravitational potential and try to describe the kinematics of NGC 5128 without inclusion of dark matter: we apply both the Newtonian and the MOND approach in the spherical approximation allowing for anisotropies in the motion of the tracers. Here, we try to answer the question whether or not it is possible to model the observed velocity dispersion of an early-type galaxy (in this case NGC 5128) without the inclusion of dark matter; the related question about the largest galactocentric radius for which dark matter is not needed is also addressed.
The plan of the paper is as follows: in Sect. 2 we present the observational data related to NGC 5128; in Sect. 3 we solve the Jeans equation in both the Newtonian and the MOND approach to determine the best-fit parameters and infer the existence of dark matter; in Sect. 4 we present our conclusions.

\section{Observational data}

The galaxy NGC 5128 (also known as the radio source Centaurus A) is the nearest large elliptical galaxy. It is the only massive elliptical in the Centaurus group (Israel 1998). The value of the distance used in this paper is $D=3.84 \pm$ $0.35 \mathrm{Mpc}$ (Rejkuba 2004). At this distance $1^{\prime} \approx 1.12 \mathrm{kpc}$, which means that $1^{\prime \prime} \approx 18.62$ pc. We adopt for the center of the galaxy the following coordinates $\left(\alpha=13^{\mathrm{h}} 25^{\mathrm{m}} 27^{\mathrm{s}} 72\right.$, $\left.\delta=-43^{\circ} 01^{\prime} 05^{\prime \prime} 8, \mathrm{~J} 2000\right)$. The effective radius is $R_{\mathrm{e}}=330^{\prime \prime}$ $(=6.15 \mathrm{kpc})$ (van den Bergh 1976) and the systemic velocity used is $v_{\text {syst }}=541 \mathrm{~km} \mathrm{~s}^{-1}$. The $B$-band luminosity of the stellar component NGC 5128 is $L_{B}=3.98 \times 10^{10} L_{\odot}$. The review of the NGC 5128 galaxy is given in the paper by Israel (1998). This galaxy is an excellent target for a detailed dynamical study because of its proximity and different available observational data: we will compare the results based on $\mathrm{PNe}$ and $\mathrm{X}$-rays. Throughout the paper we assume that the Hubble constant is $h_{0}=0.70$.

\subsection{Planetary nebulae of NGC 5128}

We use the sample of PNe from the paper by Peng et al. (2004), which contains $780 \mathrm{PNe}$. We present the kinematics of NGC 5128 based on the PNe in Table 1 and also in Fig. 1. Note that error bars are not plotted for the radial velocities: as given in Peng et al. (2004) they are taken to be $20 \mathrm{~km} \mathrm{~s}^{-1}$. The data for the PNe extend out to $78 \operatorname{arcmin}\left(=14 R_{\mathrm{e}}\right)$, which is a very large galactocentric distance that encompasses approximately the total galactic light (more precisely, 98 per cent of the total light for the de Vaucouleurs model), which is important for our study of dark matter.

The radial velocities of PNe were used to determine the kinematics of NGC 5128: we calculated the velocity dispersion, the skewness and kurtosis parameters, $s_{3}$ and $s_{4}$, which describe asymmetric and symmetric departures from the Gaussian in each bin. We used standard definitions of the skewness and kurtosis 


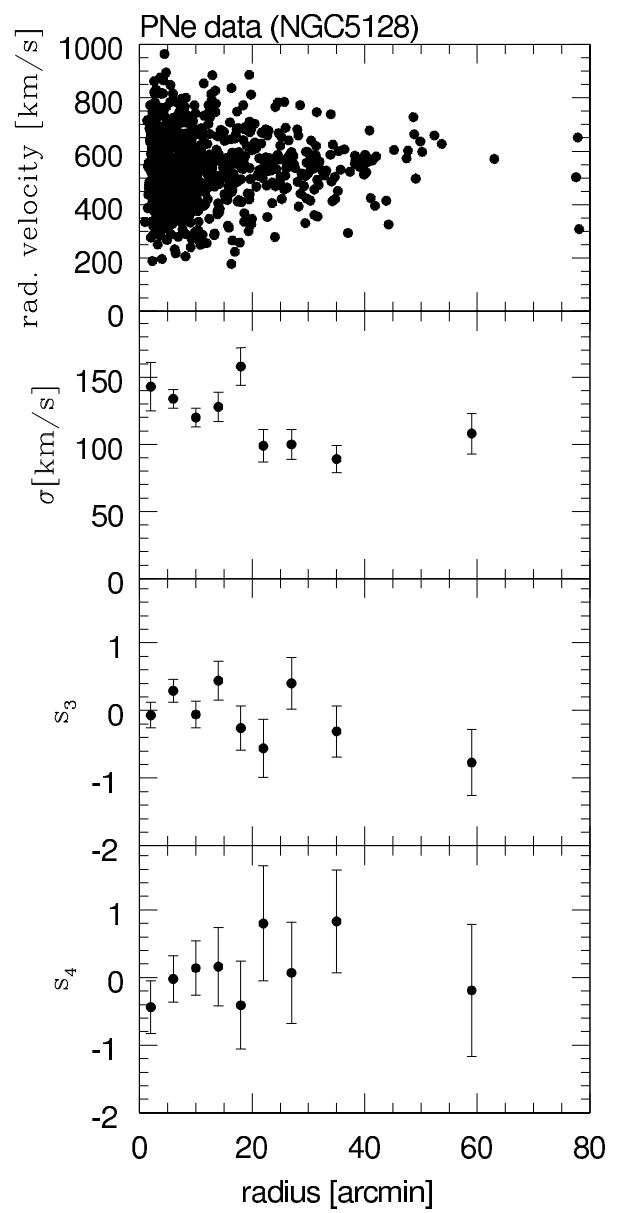

Fig. 1. Kinematics of NGC 5128 based on the sample of the PNe. From top to bottom: radial velocity of the PNe in $\mathrm{km} \mathrm{s}^{-1}$; velocity dispersion in $\mathrm{km} \mathrm{s}^{-1}$ calculated in a given bin and the $s_{3}$ and $s_{4}$ parameters, which describe symmetric and asymmetric departures from the Gaussian, respectively.

implemented in the NAG routine GO1AAF. Negative values of kurtosis $s_{4}<0$ imply tangential anisotropies and positive values of kurtosis $s_{4}>0$ imply the dominance of radial orbits. This has become a standard way of describing the anisotropies of given tracers in early-type galaxies (see for example Schuberth et al. 2009, where GCs were analyzed in the galaxy NGC 1399). From Table 1 and Fig. 1 one can see that the profile of the velocity dispersion is decreasing (apart from one anomalous point at $\sim 18$ arcmin); we also notice a mild increase of the velocity dispersion in the last bin. This will be analyzed in the next section, where we solve the Jeans equation and model the velocity dispersion of NGC 5128. The kurtotic parameter is small and it is safe to assume that there are no large anisotropies; nevertheless we will also use some anisotropic solutions in the next section to fully constrain the distribution of matter in this early-type galaxy.

For the Jeans modeling we also need the radial surface density profile of the PNe in NGC 5128. This was given in Peng et al. (2004) (see their Fig. 9), where they fitted the radial surface density with the expression $\Sigma \propto r^{-\gamma}$ and found that $\gamma=2.54$ in the interval $7 \lesssim r \lesssim 40 \mathrm{kpc}$.

\subsection{X-ray data for NGC 5128}

Kraft et al. (2003) analyzed NGC 5128 based on the results from two Chandra/ACIS-I observations and one XMM-Newton observation of X-ray emission from the interstellar medium (ISM). They found that the ISM has an average radial surface brightness profile that is well described by a $\beta$-model profile with the index $\beta=0.40 \pm 0.04$ and a temperature of $k_{B} T_{\text {ISM }}=0.29$ $\mathrm{keV}$ beyond $2 \mathrm{kpc}$ from the galactic nucleus. Kraft et al. (2003) calculated that within $15 \mathrm{kpc}$ of the nucleus the total mass of NGC 5128 is $\sim 2 \times 10^{11} M_{\odot}$. This value corresponds to the massto-light ratio $M / L_{B}=6.70$ at $15 \mathrm{kpc}\left(=2.44 R_{\mathrm{e}}\right.$, which is a region which contains 75 per cent of the total light for de Vaucouleurs law). We note that this value is very close to that inferred from the stellar content (see Sect. 3.1).

\section{Dynamical models}

For both the Newtonian and the MOND approach we solved the Jeans equation (e.g. Binney \& Tremaine 2008):

$$
\frac{\mathrm{d} \sigma_{\mathrm{r}}^{2}}{\mathrm{~d} r}+\sigma_{\mathrm{r}}^{2} \frac{\left(2 \beta_{*}+\alpha\right)}{r}=a_{\mathrm{N} ; \mathrm{M}}+\frac{v_{\mathrm{rot}}^{2}}{r}
$$

where $a_{\mathrm{N} ; \mathrm{M}}$ is an acceleration term, which is equal to $a_{\mathrm{N}}=$ $-\frac{G M(r)}{r^{2}}$ for Newtonian ("N") models and for MOND ("M") models $a_{\mathrm{M}} \mu\left(a_{\mathrm{M}} / a_{0}\right)=a_{\mathrm{N}}$ (see Sect. 3.2 for details regarding function $\mu$ ) and $\sigma_{\mathrm{r}}$ is the radial stellar velocity dispersion, $\alpha=\mathrm{d} \ln \rho / \mathrm{d} \ln r$ is the slope of tracer density $\rho$ (the surface density is given above and in the models below we use $\alpha=$ $-3.54)$. We also note that the rotation speed $v_{\text {rot }}$ of NGC 5128 is non-zero, and we take $v_{\text {rot }}=50 \mathrm{~km} \mathrm{~s}^{-1}$ (interior to 12 arcmin) and $v_{\text {rot }} \approx 100 \mathrm{~km} \mathrm{~s}^{-1}$ (beyond 12 arcmin) (Peng et al. 2004, see their Fig. 11) and is used in all modeling below. To describe the non-spherical nature of the stellar velocity dispersion a parameter $\beta_{*}$ is introduced

$\beta_{*}=1-\frac{\overline{v_{\theta}^{2}}}{\sigma_{\mathrm{r}}^{2}}$

where $\overline{v_{\theta}^{2}}={\overline{v_{\theta}}}^{2}+\sigma_{\theta}^{2}$. For $0<\beta_{*}<1$, the orbits are predominantly radial (equivalent to $s_{4}>0$ ), and the line-of-sight velocity distribution is more strongly peaked than a Gaussian profile. On the contrary, for $-\infty \leq \beta_{*}<0$ the orbits are mostly tangential (equivalent to $s_{4}<0$ ), so that the profile is more flat-topped than a Gaussian (Gerhard 1993).

For all models calculated below, we used the projected line-of-sight velocity dispersion (e.g. Binney \& Mamon 1982; Mathews \& Brighenti 2003b) given as

$\sigma_{p}^{2}(R)=\frac{\int_{R}^{r_{\mathrm{t}}} \sigma_{\mathrm{r}}^{2}(r)\left[1-(R / r)^{2} \beta_{*}\right] \rho(r)\left(r^{2}-R^{2}\right)^{-1 / 2} r \mathrm{~d} r}{\int_{R}^{r_{\mathrm{t}}} \rho(r)\left(r^{2}-R^{2}\right)^{-1 / 2} r \mathrm{~d} r}$

where the truncation radius, $r_{\mathrm{t}}$, extends beyond the observed kinematical point of the highest galactocentric radius; in all the cases $r_{\mathrm{t}}=15 R_{\mathrm{e}}$.

\subsection{Newtonian mass-follows-light models}

The case of NGC 5128 is an interesting one because this galaxy has a rather low stellar mass-to-light ratio. One may use the relation derived by Bell et al. (2003) (see their Table 7) to infer that mass-to-light ratio in the $B$-band in solar units is $M / L_{B}=6.24$ based on the color $B-V=1.00$ (value from the RC3 catalog, de Vaucouleurs et al. 1991). This value is very close to that calculated with Eq. (7) from the paper by Cappellari et al. (2006), who modeled 25 early-type galaxies out to $\sim 1 R_{\mathrm{e}}$ and found a 
Table 2. Some fits for the Newtonian constant M/L ratio and the MOND isotropic $\left(\beta_{*}=0\right)$ and anisotropic $\left(\beta_{*} \neq 0\right)$ modeling.

\begin{tabular}{lcrrl}
\hline \multicolumn{1}{c}{ Model } & Region & \multicolumn{1}{c}{$M / L_{B}$} & \multicolumn{1}{c}{$\beta_{*}$} & \multicolumn{1}{c}{$\chi^{2}$} \\
& $(2)$ & \multicolumn{1}{c}{$(3)$} & \multicolumn{1}{c}{$(4)$} & $(5)$ \\
\hline Newtonian & 1 & 6.24 & 0.00 & 0.93 \\
Newtonian & 1 & 7.00 & 0.00 & 0.47 \\
Newtonian & 2 & 6.24 & 0.00 & 3.62 \\
Newtonian & 2 & 8.00 & 0.00 & $3.32 *$ \\
Newtonian & 2 & 16.00 & 0.00 & $22.61 *$ \\
Newtonian & 1 & 6.24 & -0.10 & 0.58 \\
Newtonian & 1 & 7.50 & 0.10 & 0.44 \\
Newtonian & 2 & 6.24 & -0.10 & 3.26 \\
Newtonian & 2 & 6.24 & -0.50 & $6.09 *$ \\
MOND "simple" & 1 & 9.75 & 0.00 & 0.32 \\
MOND "simple" & 2 & 9.00 & 0.00 & 13.66 \\
MOND "simple" & 1 & 6.24 & -0.60 & 0.07 \\
MOND "simple" & 1 & 7.00 & -0.40 & 0.14 \\
MOND "simple" & 2 & 9.00 & -0.50 & 4.37 \\
MOND "standard" & 1 & 7.00 & 0.00 & 0.71 \\
MOND "standard" & 1 & 8.00 & 0.00 & 0.02 \\
MOND "standard" & 2 & 9.00 & 0.00 & 10.05 \\
MOND "standard" & 1 & 6.24 & -0.30 & 0.05 \\
MOND "standard" & 1 & 7.00 & -0.20 & 0.01 \\
MOND "standard" & 2 & 6.24 & -0.50 & 5.02 \\
MOND "toy" & 1 & 12.50 & 0.00 & 0.44 \\
MOND "toy" & 1 & 7.00 & -0.60 & 0.48 \\
MOND "toy" & 2 & 6.24 & -0.50 & 9.75 \\
MOND "toy" & 2 & 9.00 & -0.50 & 6.10 \\
\hline
\end{tabular}

Notes. Column (1): the name of the model; Col. (2): region for which the fit is calculated; " 1 " is for the innermost region ( $r \leq 10$ arcmin) and "2" is for the outer region ( $r>10 \mathrm{arcmin})$; Col. (3): mass-to-light ratio in the $B$-band used; Col. (4): the best-fitting value of the $\beta_{*}$ parameter; Col. (5): reduced $\chi^{2}$ of the fit for a given model (see text for details).

tight correlation between the mass-to-light ratio and the velocity dispersion. This equation in the $B$-band gives the following value for NGC 5128: $M / L_{B}=6.39 \pm 0.24$ (here we used $\sigma_{\mathrm{e}}=140 \mathrm{~km} \mathrm{~s}^{-1}$ for the value of the velocity dispersion within one effective radius).

Since we deal in this work with constant mass-to-light ratio models, we considered relations that include the stellar mass distributed in the form of the standard Hernquist (1990) profile

$\rho_{\mathrm{H}}(r)=\frac{M_{T}}{2 \pi} \frac{a}{r} \frac{1}{(r+a)^{3}}$,

which has two parameters: the total mass $M_{\mathrm{T}}$ and scale length, $a$, where $R_{\mathrm{e}}=1.8153 a$. When necessary, i.e. when we encountered the need to have a mass-to-light ratio higher than that obtained with only a stellar component in order to fit the observations, we assumed that the distribution of hypothetical dark matter will also obey this relation. The characteristic radius, $a$, in this case, is the same as for stars.

We solved the Jeans Eq. (1) taking into account both isotropic $\left(\beta_{*}=0\right)$ and anisotropic $\left(\beta_{*} \neq 0\right)$ cases always assuming spherical symmetry. The isotropic cases are obviously used here as a computational convenience, because in the regions where rotational velocity is significant, the $\beta_{*}$ parameter should be strongly negative. The results are given in Table 2 , where we present the best-fit values for some models for the mass-to-light ratio and the associated $\chi^{2}$ values. The rotational velocity of NGC 5128 varies with the radius; as mentioned above we therefore divide the galaxy into two regions: the interior region $\left(r \leq 10\right.$ arcmin) where we take $v_{\text {rot }}=50 \mathrm{~km} \mathrm{~s}^{-1}$ (this is obviously a simplification, see Fig. 10 from the paper by Peng et al. 2004) and the exterior region ( $r>10$ arcmin) where the velocity is approximately constant and equal to $v_{\text {rot }}=100 \mathrm{~km} \mathrm{~s}^{-1}$. Note that beyond 10 arcmin the $\chi^{2}$ values are rather high due to a sudden increase of the velocity dispersion at $\sim 18$ arcmin. The results related to the successful fits of the outermost point are marked with an asterisk and are characterized by higher values of $\chi^{2}$.

The best fit assuming an isotropy interior to $\sim 35$ arcmin was obtained for a mass-to-light ratio: $6.24 \lesssim M / L_{B} \lesssim 7.00$, which agrees with a value inferred from the stellar component. This clearly means that dark matter does not necessarily have to extend out to $\sim 35 \operatorname{arcmin}\left(\sim 6.4 R_{\mathrm{e}}\right)$ if we assume isotropy. Beyond $\sim 35$ arcmin the mass-to-light ratio needs to increase if we are to fit the outermost point: in Fig. 2 we show two values, $M / L_{B}=8.00$ and $M / L_{B}=16.00$, which roughly cover the whole permitted range; both values assume the existence of the unseen mass component, small in the first case ( $\sim 20$ per cent) and significant in the second case (a dark component $\sim 2.5$ times larger than the stellar one).

Although the testing of different dark matter halos is not the aim of this paper, we decided to test two additional dark matter profiles (the one based on the Hernquist profile was already tested above). To infer the influence of dark matter we added to the mass generated by the stellar component $\left(M / L_{B}=6.24\right)$ two different dark halos. In Fig. 2 we also show two fits obtained with two different models of dark halo for isotropic orbits. The dashed line is obtained for the Burkert (1995) model with the following parameters: $\rho_{0}=0.02 M_{\odot} \mathrm{pc}^{-3}$ and $r_{0}=541$ arcsec $(\approx 10 \mathrm{kpc})$. One can see that the outermost point can be fitted implying $M / L_{B} \gtrsim 11$ there; the points between 10 and 40 arcmin are not successfully fitted (the quality of the fit between 10 and 60 arcmin is characterized by $\chi_{\mathrm{B}}^{2}=3.13$ ). The dot-dashed line is for the NFW (Navarro, Frank \& White 1997) halo with $\rho_{\mathrm{s}}=0.02 M_{\odot} \mathrm{pc}^{-3}$ and $r_{\mathrm{s}}=771.41 \operatorname{arcsec}(\approx 14.4 \mathrm{kpc})$. The outermost point is well fitted (implying $M / L_{B} \sim 12$ there); the points between 10 and 40 arcmin are again not successfully fitted and the discrepancies are even larger than for the Burkert model (the quality of the fit between 10 and 60 arcmin is characterized by $\chi_{\mathrm{NFW}}^{2}=5.12$ ). Numerous other dark matter models are obviously possible and we refer the reader to the paper by Bullock et al. (2001), where various dark matter halo density profiles are studied in detail.

Since $\beta_{*}$ is essentially a free parameter in our study, we searched for the best value of $\beta_{*}$ for the value of the mass-tolight ratio inferred from the color of NGC $5128\left(M / L_{B}=6.24\right)$. Therefore, when we solved the Jeans equation for the anisotropic case, we studied the cases for which $-0.5 \leq \beta_{*} \leq 0.5$, thus allowing for moderate anisotropies as hinted by observations. The results are shown in Fig. 3. In the innermost part $(r \leq 10)$ arcmin a fit with $M / L_{B}=6.24$ and $\beta_{*}=-0.10$ can provide an agreement to the observed velocity dispersion. Several fits with the massto-light ratio in the interval $5.75 \leq M / L_{B} \leq 7.50$ with moderate anisotropies provide good agreement with the observed velocity dispersion in the inner region (out to $\sim 35$ arcmin) (see Fig. 3 and Table 2 for details). To fit the outermost point at 59 arcmin we used the mass-to-light ratio inferred from the stellar component $\left(M / L_{B}=6.24\right)$ and imposed tangential anisotropies $\left(\beta_{*}=-0.5\right)$ to avoid the inclusion of dark matter (the alternative is given earlier when we fitted this point without anisotropies but with dark matter).

To summarize the conclusions for the Newtonian approach: the observed velocity dispersion of NGC 5128 can be described without the need of dark matter out to $\sim 6.4 R_{\mathrm{e}}$ (=35 arcmin; a region that encompasses $\sim 92$ per cent of the total light for the 


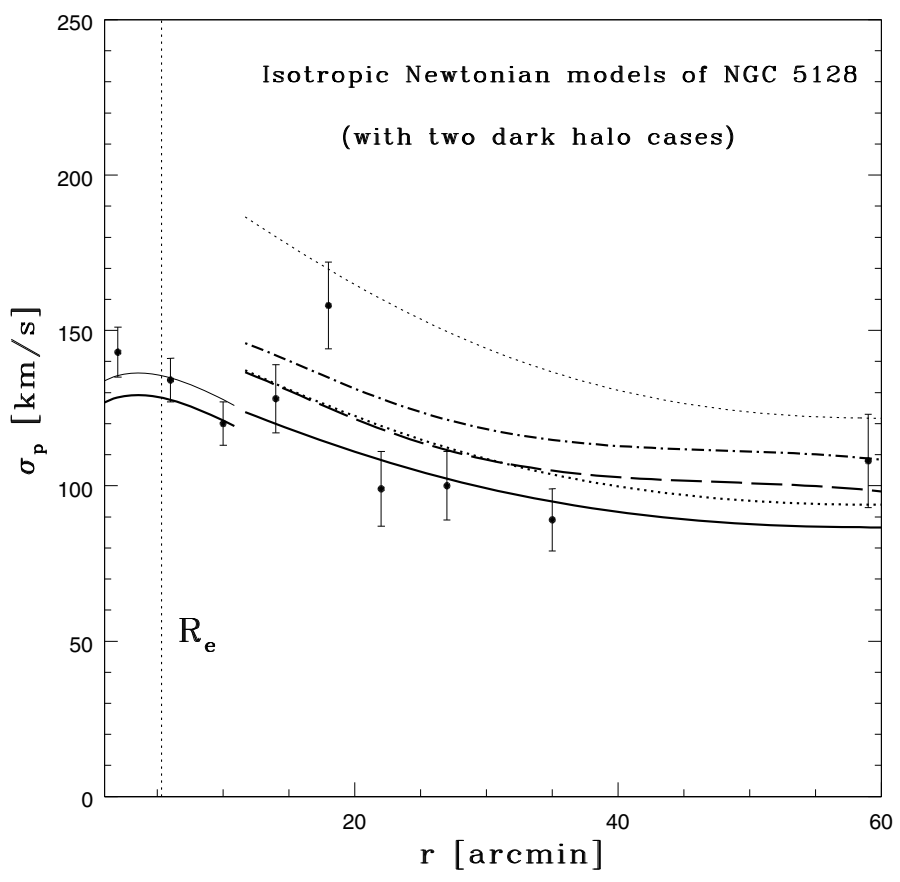

Fig. 2. Isotropic $\left(\beta_{*}=0\right)$ Jeans modeling of the projected velocity dispersion of NGC 5128 using Newtonian mass-follows-light models. Interior part $(r \leq 10 \mathrm{arcmin})$ : thick solid line is obtained for $M / L_{B}=6.24$ and thin solid line is for $M / L_{B}=7.00$. Exterior part ( $r>10$ arcmin): thick solid line is for $M / L_{B}=6.24$, thick dotted line is for $M / L_{B}=8.00$ and thin dotted line is for $M / L_{B}=16.00$. Dotted vertical line here and in all following figures denotes one effective radius. Two additional fits which include dark matter halos are also shown: the dashed line is for the Burkert halo and the dot-dashed line is for the NFW halo. See text for details.

de Vaucouleurs law). This is the largest galactocentric distance in an early-type galaxy for which dark matter is not necessary to explain its internal kinematics. The obtained value of the massto-light ratio $\left(6.24 \lesssim M / L_{B} \lesssim 7.00\right)$ agrees with the value inferred from the X-rays (Kraft et al. 2003). In the outer region of NGC 5128, $7<r<14 R_{\mathrm{e}}(=78$ arcmin) we encountered an increasing velocity dispersion, which implies an increase of the mass-to-light ratio (or tangential anisotropies) implying certain amounts of dark matter in these regions (if any). An important remark is necessary at this point: as the $\gamma$ parameter, which was calculated by Peng et al. (2004), is valid for the region interior to $\sim 40$ arcmin, any conclusion regarding both isotropic and anisotropic Newtonian models pertaining to the outermost region (beyond $\sim 40$ ) arcmin must therefore be taken with a great caution (the same remark is obviously also valid for the MOND modeling).

\subsection{MOND models}

In our MOND models we use three different formulas: (i) the "simple" MOND formula from Famaey \& Binney (2005); (ii) the "standard" formula (Sanders \& McGaugh 2002); and (iii) the Bekenstein's "toy" model (Bekenstein 2004). The Newtonian acceleration is given as $a_{\mathrm{N}}=a \mu\left(a / a_{0}\right)$, where $a$ is the MOND acceleration. As usual, $\mu(x)$ is the MOND interpolating function, where $x \equiv a / a_{0}$, and $a_{0}=1.35_{-0.42}^{+0.28} \times 10^{-8} \mathrm{~cm} \mathrm{~s}^{-2}$ is a universal constant (Famaey et al. 2007). For $a \gg a_{0}$ the interpolation function $\mu\left(a / a_{0}\right) \approx 1$ and the Newtonian relation is recovered; for $a \ll a_{0}$ we have $\mu=a / a_{0}$. It can be shown

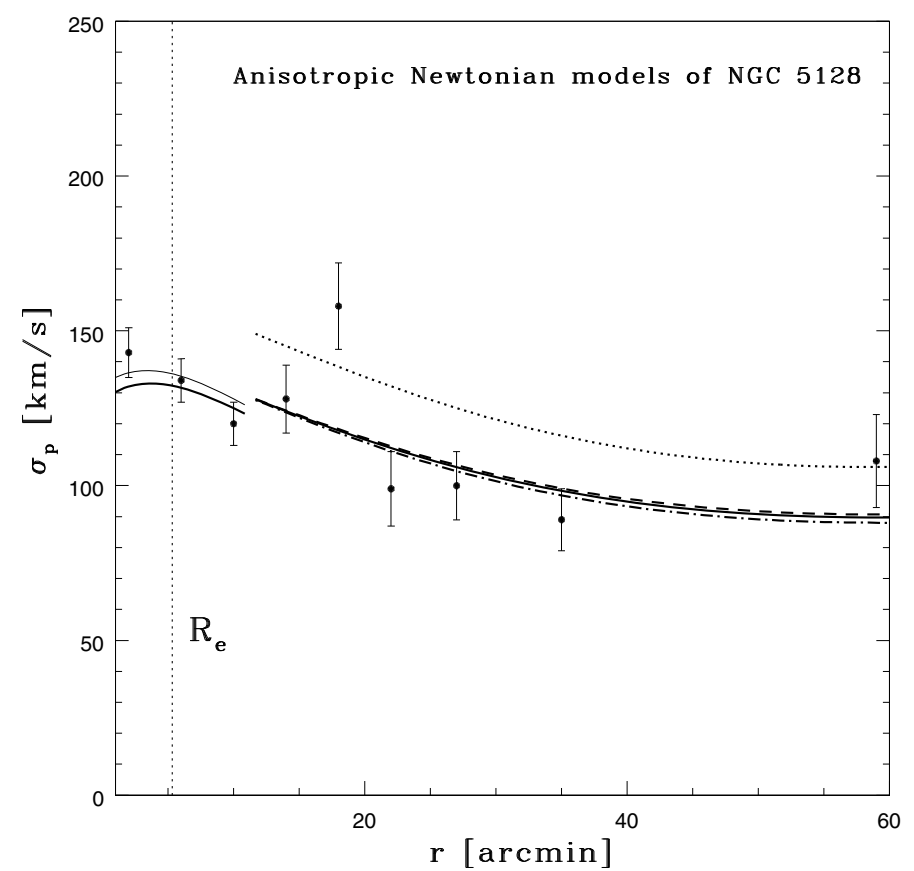

Fig. 3. Anisotropic $\left(\beta_{*} \neq 0\right)$ Jeans modeling of projected velocity dispersion of NGC 5128 using Newtonian mass-follows-light models. Interior part $(r \leq 10 \mathrm{arcmin})$ : thick solid line is obtained for $M / L_{B}=6.24$ and $\beta_{*}=-0.10$ and thin solid line is for $M / L_{B}=7.50$ and $\beta_{*}=0.10$. Exterior part $(r>10$ arcmin $)$ : thick solid line is obtained for $M / L_{B}=$ 6.24 and $\beta_{*}=-0.10$, dashed line is for $M / L_{B}=5.75$ and $\beta_{*}=-0.20$, dot-dashed is for $M / L_{B}=7.25$ and $\beta_{*}=0.10$. Thick dotted line is for $M / L_{B}=6.24$ and $\beta_{*}=-0.50$.

(e.g. Angus et al. 2008) that the MOND dynamical mass, $M_{\mathrm{M}}$, can be expressed in terms of the Newtonian mass, $M_{\mathrm{N}}$ through

$M_{\mathrm{M}}(r)=M_{\mathrm{N}}(r) \times \mu(x)$.

We assume different forms of the interpolation function for different MOND models (the expressions for the circular velocities are given in Samurović \& Ćirković 2008).

1. A "simple" MOND formula is given by

$$
\mu(x)=\frac{x}{1+x} .
$$

2. A "standard" MOND formula is given by

$$
\mu(x)=\frac{x}{\sqrt{1+x^{2}}} .
$$

3. Finally, for the "toy" model the MOND formula is given as

$$
\mu(x)=\frac{-1+\sqrt{1+4 x}}{1+\sqrt{1+4 x}} .
$$

We tested all three MOND models using the Jeans models assuming both an isotropic and anisotropic case in the spherical approximation using different dynamical masses for three different MOND models. In Fig. 4 (Fig. 5) we show some fits for isotropic (anisotropic) models. The $\chi^{2}$ values for the best fits are given in Table 2 (the values pertaining to the fits of the outermost point are not given, but they are high).

Again we divided the galaxy into two regions: the interior region $\left(r \leq 10 \operatorname{arcmin}\right.$ and $\left.v_{\text {rot }}=50 \mathrm{~km} \mathrm{~s}^{-1}\right)$ and the exterior region $\left(r>10\right.$ arcmin and $\left.v_{\text {rot }}=100 \mathrm{~km} \mathrm{~s}^{-1}\right)$. Only the "standard" MOND model with $\left(7 \lesssim M / L_{B} \lesssim 8\right)$ may provide 


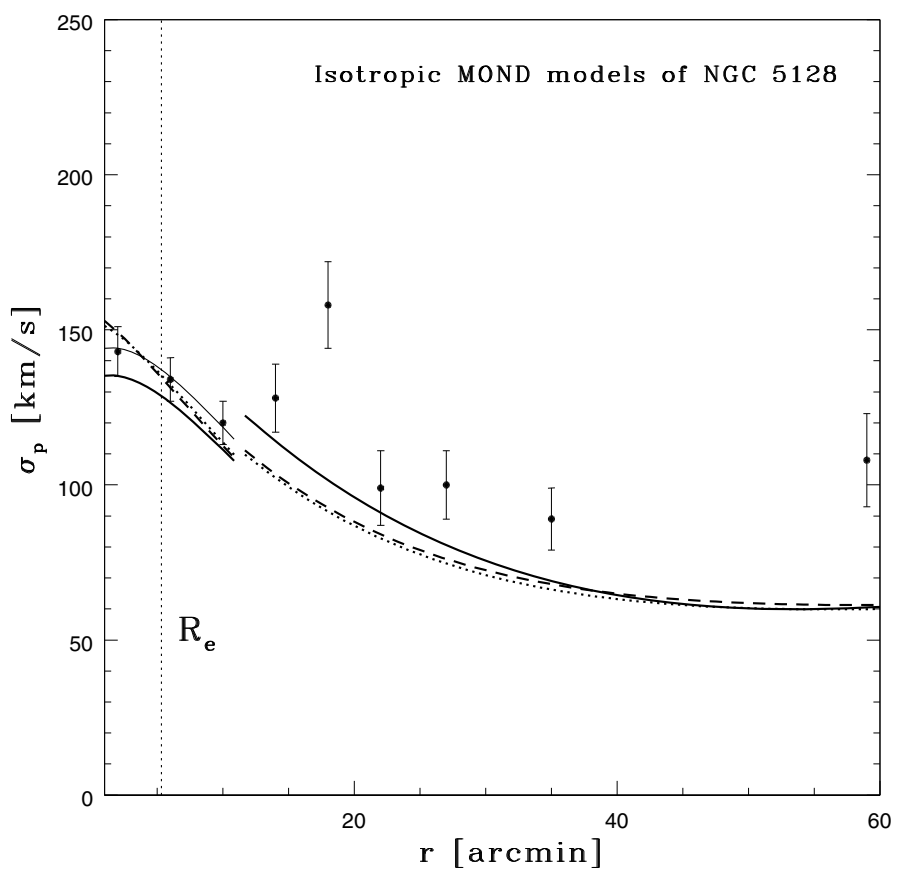

Fig. 4. Isotropic $\left(\beta_{*}=0\right)$ MOND models of the projected velocity dispersion of NGC 5128. Interior part ( $r \leq 10 \operatorname{arcmin})$ : the solid lines are for the "standard" model: the thick line is for $M / L_{B}=7.00$ and the thin line is for $M / L_{B}=8.00$. The thick dotted line is for the "simple" model, for which $M / L_{B}=9.75$. The thick dashed line is for the "toy" model, for which $M / L_{B}=12.50$. Exterior part $(r>10 \operatorname{arcmin})$ : the solid line is for the "standard" model, for which $M / L_{B}=9.00$. The dotted line is for the "simple" model, for which $M / L_{B}=9.00$. The dashed line is for the "toy" model, for which $M / L_{B}=12.00$.

a good fit without anisotropy. Both the "simple" and the "toy" models require much larger mass-to-light ratios in this region (see Fig. 4). For the isotropic case we found that the "standard" MOND model with the mass-to-light ratio inferred from the stellar content $\left(M / L_{B}=6.24\right)$ again provides better fits between 10 and 40 arcmin than the "simple" and "toy" models (with the same mass-to-light ratio), but it is still far from perfect (not shown, but presented in Table 2). If one wants to fit points beyond $\sim 10$ arcmin in the "standard" model one needs to increase the mass-to-light ratio: a fit for which $M / L_{B}=9.00$ is shown in the same figure. Even this high value (which includes a certain amount, $\sim 30$ percent of unseen matter) cannot provide a fit for the points in the interval $30 \lesssim r<40$ arcmin. For the "simple" and "toy" models the increase of the mass-to-light ratio to 9.00 and 12.00 respectively does improve the fits: the points interior to $\sim 10$ arcmin are fitted, but those beyond $\sim 10$ arcmin cannot be fitted, which implies even higher values of the mass-to-light, meaning that more unseen (dark) matter is necessary in those regions. None of the three isotropic MOND models can provide a fit for the outermost point with stellar mass-to-light ratio, which suggests that in order to fit the outermost point one needs to assume anisotropy of the tracers $(\mathrm{PNe})$.

In Fig. 5 we present different anisotropic MOND models; our goal again was to find acceptable models that would provide reasonable fits in both inner $(0<r<40$ arcmin $)$ and outer ( $r>40$ arcmin) regions. In the innermost region ( $r \leq 10 \mathrm{arcmin})$ we found that $6.24 \lesssim M / L_{B} \lesssim 7.00$ and various tangential anisotropies may provide a good fit to the observed velocity dispersion. From Fig. 5 one can see that "standard" MOND models based on the mass-to-light ratio inferred from the stellar content $\left(M / L_{B}=6.24\right)$ provide a satisfactory fit for the whole

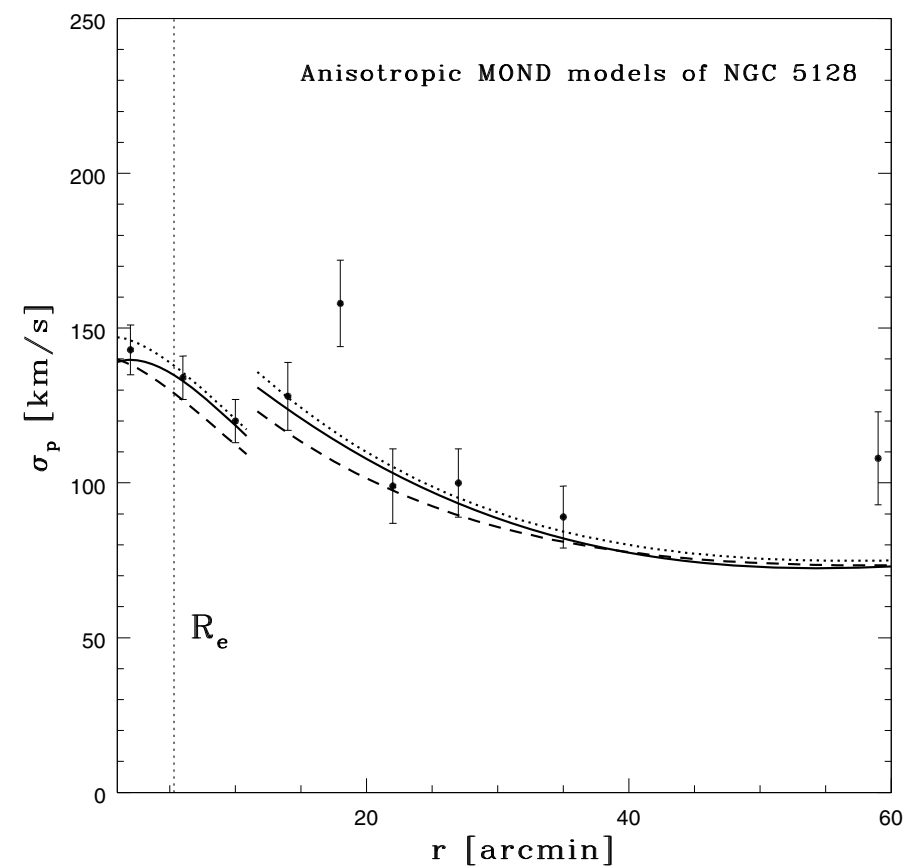

Fig. 5. Anisotropic $\left(\beta_{*} \neq 0\right)$ MOND models of the projected velocity dispersion of NGC 5128. Interior part $(r \leq 10 \mathrm{arcmin})$ : the solid line is obtained for the "standard" model with $M / L_{B}=6.24$ and $\beta_{*}=-0.30$. The dotted line is obtained for the "simple" model, for which $M / L_{B}=6.24$ and $\beta_{*}=-0.60$. The dashed line is for the "toy" model, for which $M / L_{B}=7.00$ and $\beta_{*}=-0.60$. Exterior part $(r>10$ arcmin): the solid line is obtained for the "standard" model, for which $M / L_{B}=6.24$ and $\beta_{*}=-0.50$. The dotted line is for the "simple" model, for which $M / L_{B}=9.00$ and $\beta_{*}=-0.50$. The dashed line is for the "toy" model, for which $M / L_{B}=9.00$ and $\beta_{*}=-0.50$.

region interior to $\sim 40$ arcmin: the model with a moderate tangential anisotropy $\left(\beta_{*}=-0.30\right)$ provides a fit interior to $\sim 10 \mathrm{arcmin}$ and the model with a stronger tangential anisotropy $\left(\beta_{*}=-0.50\right)$ provides a fit for $10 \lesssim r \lesssim 40 \mathrm{arcmin}$. This is the unique case we have encountered in the MOND modeling when one does not need dark matter to successfully fit the observed velocity dispersions of NGC 5128 out to $\sim 40$ arcmin. The MOND "simple" (with $M / L_{B}=6.24$ ) and "toy" (with $M / L_{B}=7.00$ ) models with significant tangential anisotropies can provide a fit in the innermost region (interior to $\sim 10 \operatorname{arcmin} ; \beta_{*}=-0.60$; see Fig. 5) and beyond ( $10 \lesssim r \lesssim 40$ arcmin; with $\left.\beta_{*}=-0.50\right)$ for $M / L_{B}=9.00$ (both models). As for the isotropic case it is obvious that in order to fit the observed data in the region between 10 and 40 arcmin one needs at least 30 per cent of dark matter for both the "simple" and "toy" models. We again could not obtain successful fits of the observed velocity dispersion in the last bin; to do this one needs $M / L_{B} \gtrsim 30$ and significant tangential anisotropies $\left(\beta_{*} \lesssim-0.50\right)$. However, regarding the fit of the outermost point we repeat our earlier observation with regard to Newtonian models because it is still valid: fits obtained with $\gamma=2.54$ are uncertain because this parameter was obtained in the region beyond $\sim 40$ arcmin.

The overall conclusion regarding MOND models is that interior to $\sim 40$ arcmin only a "standard" MOND model with tangential anisotropies can provide a successful fit of the observed velocity dispersion without the need of dark matter. Both the "simple" and "toy" models need at least 30 per cent of dark matter to obtain a fit in this region. 


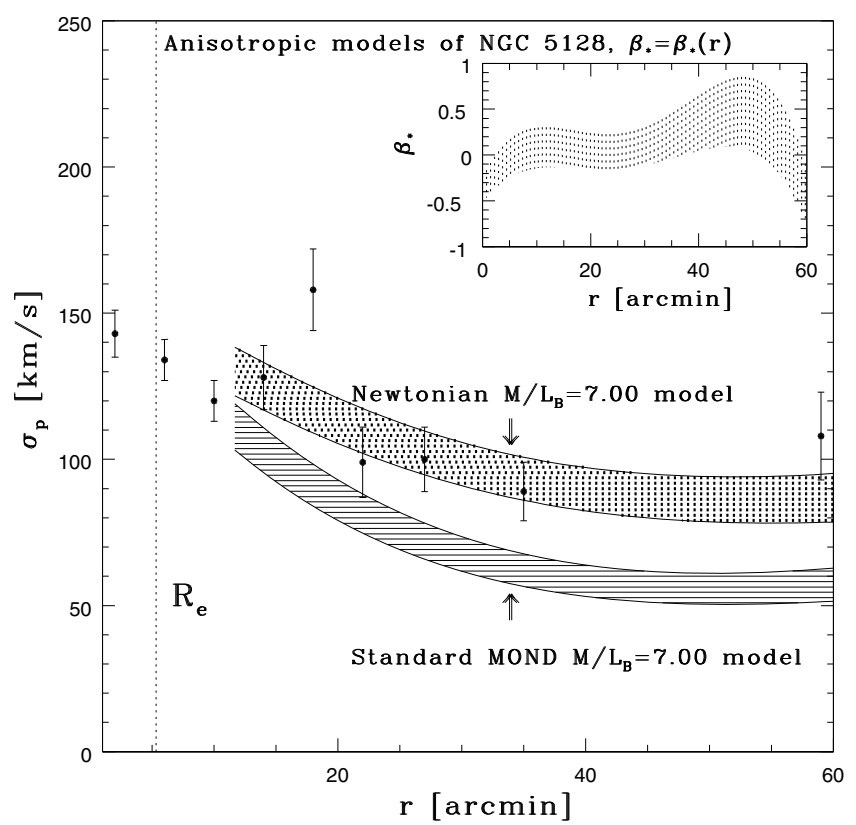

Fig. 6. Anisotropic modeling of NGC 5128 with $\beta_{*}=\beta_{*}(r)$. The Newtonian mass-follows-light and "standard" MOND models are presented for $M / L_{B}=7$. The inserted box shows the allowed range of values of the $\beta_{*}$ parameter for our models. See text for details.

\subsection{Newtonian and MOND models with a radially varying $\beta_{*}$-parameter}

We follow here the recommendation of the referee and model the galaxy NGC 5128 using a $\beta_{*}$ parameter that is not constant and varies with the radius: we use $\beta_{*}$ based on the measured values of the $s_{4}$ parameter (a similar approach was used when we did the modeling of IC 1459 and NGC 3379 in Samurović \& Danziger 2005). We repeat here that the estimates of anisotropy are very rough (and therefore produce large error bars) and hope that future observations of NGC 5128 will provide a larger sample of tracers and therefore provide more accurate estimates of anisotropies. We based our models on the estimates of the $s_{4}$ parameter using the whole range of accepted values given by the error bars of this parameter in Table 1; in the inserted box of Fig. 6 we show the allowed range of values of the $\beta_{*}$ parameter for our models. Because we are interested in dark matter we modeled only the exterior part ( $r>10 \mathrm{arcmin})$, and for both approaches, Newtonian and MOND, we used $M / L_{B}=7$, implying a lack of dark matter. We tested all three MOND models, but present only the results based on the "standard" model, because it produced a marginally better fit than the other two MOND models (but far from good). From Fig. 6 it is evident that the Newtonian model with realistic anisotropies may provide a good fit interior to $\sim 40$ arcsec $\left(\sim 8 R_{\mathrm{e}}\right)$; it can even marginally fit the outermost point at 59 arcmin. On the other hand the MOND models (the "standard" model as their representative in this case) completely fail to fit the observed velocity dispersion beyond $\sim 2 R_{\mathrm{e}}$. We repeat that to fit the observed velocity dispersion (interior to $\sim 40$ arcmin) we need to use ad hoc anisotropic MOND models with tangential anisotropies (see Fig. 5).

\section{Conclusions}

We used PNe kinematics of the well-known early-type galaxy NGC 5128 to model the observed velocity dispersions out to $\sim 59 \operatorname{arcmin}\left(=10.7 R_{\mathrm{e}}\right)$. We solved the Jeans equations for various Newtonian mass-follows-light and MOND models assuming both isotropy and anisotropy in the spherical approximation in order to study the existence of dark matter in this early-type galaxy. Our conclusions are as follows:

1. We studied the kinematics of NGC 5128 and found that the velocity dispersion decreases from $143 \pm 18 \mathrm{~km} \mathrm{~s}^{-1}$ near the center to $89 \pm 10 \mathrm{~km} \mathrm{~s}^{-1}$ at $35 \operatorname{arcmin}\left(=6.4 r_{\mathrm{e}}\right)$ and then increases at $59 \operatorname{arcmin}\left(=10.7 r_{\mathrm{e}}\right)$ to $108 \pm 15 \mathrm{~km} \mathrm{~s}^{-1}$. We calculated both skewness and kurtosis, which are not significant, which implies that the orbits of PNe in NGC 5128 are close to isotropic. Nevertheless, in our models we allowed for certain anisotropies.

2. We solved the Jeans equation for the Newtonian massfollows-light models assuming both isotropy and anisotropy in the spherical approximation. We found that assuming isotropy successful fits interior to $\sim 35$ arcmin were obtained for $6.24 \lesssim M / L_{B} \lesssim 7.00$, which agrees with a value inferred from the stellar component and also with the X-rays (out to 15 arcmin). To fit the outermost point at 59 arcmin in the Newtonian mass-follows-light models one needs either additional unseen (dark) matter or tangential anisotropies, although the fit of this point is marginally consistent with the observations when we assume a radially varying anisotropy of the form $\beta_{*}=\beta_{*}(r)$ based on the observed anisotropies. In any case, it is safe to conclude that interior to $\sim 6.4 R_{\mathrm{e}}$ one does not need dark matter to explain the observed velocity dispersion of NGC 5128. This is the largest galactocentric distance in any early-type galaxy for which dark matter is not necessary to explain its internal kinematics.

3. We also solved the Jeans equation for various MOND models, again assuming both isotropy and anisotropy in the spherical approximation. No isotropic MOND model without dark matter could provide a successful fit interior to $\sim 40 \mathrm{arcmin}$. For the anisotropic MOND models interior to $\sim 40$ arcmin only the "standard" MOND model with tangential anisotropies can provide a successful fit of the observed velocity dispersion without the need of dark matter (using $M / L_{B}=6.24$ and $-0.1 \lesssim \beta_{*} \lesssim-0.5$ ). Both the "simple" and "toy" models need at least $\sim 30$ per cent of dark matter to obtain a fit in this region. No anisotropic MOND model with low values of the mass-to-light ratio (i.e. based on the stellar content only) could fit the outermost region of NGC 5128. No MOND model with radially varying anisotropy based on the observations, $\beta_{*}=\beta_{*}(r)$, could fit the regions beyond $\sim 10$ arcmin.

Acknowledgements. The author thanks M.M. Ćirković for numerous interesting discussions. This work was supported by the Ministry of Science of the Republic of Serbia through the project no. 146012, "Gaseous and stellar component of galaxies: interaction and evolution". This research made use of the NASA/IPAC Extragalactic Database (NED), which is operated by the Jet Propulsion Laboratory, California Institute of Technology, under contract with the National Aeronautics and Space Administration. We acknowledge the usage of the HyperLeda database (http: //leda . univ-lyon 1 . fr). The author gratefully acknowledges the valuable comments of the anonymous referee, which helped to improve the quality of the manuscript.

\section{References}

Angus, G. W., Famaey, B., \& Buote, D. A. 2008, MNRAS, 387, 1470 Bekenstein, J. 2004, Phys. Rev. D, 70, 083509

Bell, E. F., McIntosh, D. H., Katz, N., \& Weinberg, M. D., ApJSS, 149, 289

Binney, J. J., \& Mamon, G. 1982, MNRAS, 200, 361

Binney, J. J., \& Tremaine, S. 2008, Galactic Dynamics, 2nd edn. (Princeton: Princeton Univ. Press) 
Bullock, J. S., Kolatt, T. S. Sigad, Y. et al. 2001, MNRAS, 321, 559

Burkert, A. 1995, ApJ, 447, L25

Cappellari, M., Bacon, R., Bureau, M. et al. 2006, MNRAS, 366, 1126

de Vaucouleurs, G., de Vaucouleurs, A. Corwin, H. G. Jr., et al. 1991, Third Reference Catalogue of Bright Galaxies (New York: Springer-Verlag)

Dekel, A., Stoehr, F., Mamon, G. A., Cox, T. J. \& Primack, J. R. 2005, Nature, 437, 707

Diehl, S., \& Statler, T. S. 2007, ApJ, 668, 150

Famaey, B., \& Binney, J. 2005, MNRAS, 363, 603

Famaey, B., Gentile, G., Bruneton, J.-P., \& Zhao, H. S. 2007, Phys. Rev. D, 75, 063002

Gerhard, O. 1993, MNRAS, 265, 213

Hernquist, L. 1990, ApJ, 356, 359

Israel, F. P. 1998, A\&A R, 8, 237

Kraft, R. P., Forman, W. R., Jones, C., et al. 2003, ApJ, 592, 129

Łokas, E. W. 2008, MNRAS, 680, L101

Mathews, W. G., \& Brighenti, F. 2003a, ARA\&A, 41, 191

Mathews, W. G., \& Brighenti, F. 2003b, ApJ, 599, 992

Mathieu, A., Dejonghe, H., \& Hui, X. 1996, A\&A, 309, 30

Milgrom, M. 1983, ApJ, 270, 365
Navarro, J. F., Frenk, C. S. \& White, S. D.M. 1997, ApJ, 490, 493

Peng, E. W., Ford, H. C., \& Freeman, K. C. 2004, ApJ, 602, 685

Rejkuba, M. 2004, A\&A, 413, 903

Richtler T., Dirsch B., Gebhardt K., et al. 2004, AJ, 127, 2094

Richtler, T, Schuberth, Y., Hilker, M., et al. 2008, A\&A, 478, L23

Romanowsky, A. J., Douglas, N. G., Arnaboldi, M., et al. 2003, Science, 5640, 1696

Samurović, S. 2007, Dark Matter in Elliptical Galaxies, Publications of the Astronomical Observatory of Belgrade, No. 81.

Samurović, S., \& Danziger, I. J. 2005, MNRAS, 363, 769

Samurović, S., \& Danziger, I. J. 2006, A\&A, 458, 79

Samurović, S., \& Ćirković M. M. 2008, A\&A, 488, 873

Sanders, R. H., \& McGaugh, S. 2002, ARA\&A, 40, 263

Schuberth, Y., Richtler, T., Hilker, M., et al. 2010, A\&A, 513, A52

Tiret, O., Combes, F., Angus, G. W., Famaey, B., \& Zhao, H. S. 2007, A\&A, 476, L1

Tonry, J. L. 1983, ApJ, 266, 58

van den Bergh, S. 1976, ApJ, 208, 673

van der Marel, R. P. 1991, MNRAS, 253, 710

Weijmans, A., Cappellari, M., Bacon, R. et al. 2009, MNRAS, 398, 561 